\title{
Erzeugung von Cordierit-Keramikschäumen über die Zwischenstufe hochgefüllter Polyurethan-Schaumstoffe
}

\author{
Michael Herzog, Rainer Wegner
}

\begin{abstract}
Zusammenfassung
Der Beitrag berichtet von der Entwicklung von leichten keramischen Massen mit speziellen Schaumstrukturen. Hierzu wurden geeignete Ausgangsstoffe ermittelt sowie ein Verfahren entwickelt, das seine Leistungsfähigkeit mit Keramikformkörpern demonstriert hat.

Die Arbeiten beinhalteten im Kern die Entwicklung eines neuartigen Verfahrens der Herstellung keramischer Schaumstoffe mittels PUR-Systemen auf der Basis von Recyclatpolyolen, wobei die keramischen Pulver (u. a. aufbereitete Schlacken und Cordierit-Keramik aus dem Recycling von Abgas-Katalysatoren) in niedrigviskose Polyole eingebracht und anschließend mit Isocyanaten zu anorganisch-organischen Verbundschaumstoffen geschäumt wurden. Die organischen Bestandteile wurden anschließend ausgebrannt und zuletzt die Keramik gesintert. An den erhaltenen Formkörpern wurden mechanische Kennwerte bestimmt, die Strukturen mikroskopisch charakterisiert und Gebrauchswerteigenschaften erfasst.

Die entwickelten keramischen Schaumstoffe können als in der Kälte formbeständige Körper für entsprechend belastete Bauteile herangezogen werden und eignen sich zudem als Trägermaterialien für Katalysatoren oder für Aufgaben im Bereich der Filtration.
\end{abstract}

\section{Abstract}

In this paper we report on the development of light ceramic foams of special structures. To this end, suitable raw materials and processing parameters were established to demonstrate the properties and performance of the products.

The investigation incorporates the development of a new process to produce foamed ceramics by means of PUR systems based on recycling polyols. Thus, the ceramic powders (amongst others processed slag and cordierite originating from the recycling of car catalysts) were mixed with low viscosity polyols and foamed by the reaction with isocyanates forming structured inorganic-organic composites. In the following step, the organic component was burned out and the ceramic materials simultaneously sintered. Mechanical properties of the resulting ceramic bodies were determined, structures were investigated by microscopicy, and properties for technical use received. The ceramic foams produced are useful to serve as dimensionally stable bodies for low temperature use for special parts in cryogenics, as a substrate for biocatalysts, as precious metal free catalysts in Diesel engine exhaust, or for tasks in the field of filtration.

\section{$1 \quad$ Einleitung}

Schaumstoffe genießen als zelluläre Festkörper aus Polymeren, Metallen oder Keramiken seit geraumer Zeit ein erhöhtes Interesse in den Werkstoffwissenschaften [1]. Sie bieten gegenüber massiven Materialien neben Gewichtsvorteilen ein interessantes, prinzipiell mit den mikroskopischen räumlichen Strukturen variierbares Eigenschaftsspektrum [2].

Keramikschaumstoffe werden heute auf vielen Gebieten eingesetzt, z. B. als Hochtemperaturisolationen [3], als Trägermaterial in der Biotechnologie [4], als Membranen zur Gas- oder Flüssigkeitstrennung bzw. -reinigung oder als Träger für Katalysatoren in der chemischen Industrie.

Die Herstellung kann nach verschiedenen Technologien erfolgen:

- Aufschäumen von keramischen Massen durch Gase oder Dämpfe und Brennen der keramischen Massen nach klassischen Brennverfahren [5],
- Tränken von vorgebildeten Schaumstoffen mit Aufschlämmungen keramischer Massen und Verdampfen des Schaumgerüsts [6],

- Mischen von keramischen Massen und organischem Material (Stärke, Holzmehl, Kunststoffe) zu einem Formkörper und Verdampfen des organischen Materials,

- Verschäumung von keramischen Pulvern mit organischen Reaktionssystemen (z. B. zur Erzeugung von Polyurethanen) zu hoch feststoffbeladenen polymeren Schaumstoffen, die oxidiert und gesintert werden [7]. Mittels weiterer Nachbehandlungen, z. B. Ausfüllen hohler Stege durch Tauchung primärer Schaumkeramiken in flüssigem Silizium wurden gezielt weitere Eigenschaftsverbesserungen verfolgt [8].

Wesentlich aufwändiger sind anspruchsvolle Laborverfahren wie die Pyrolyse von geschäumten Siloxanen, die mit $\mathrm{Si} / \mathrm{SiC}$ bzw. $\mathrm{SiO}_{2}$ (Quarz) gefüllt sind [9].

Schaumkeramiken wurden als Basismaterial für MMC (Metall-Matrix-Composits) untersucht [10], um 
anschließend mit flüssigen Metallen ausgegossen zu werden und im erstarrten Zustand eine Keramikverstärkung für Metalle zu bieten. Hierdurch wurde auf eine erhöhte Abriebfestigkeit von Metallen, Festigkeitserhöhung von Leichtmetallen oder den verbesserten Wärmetransport in mikroelektronischen Substraten gezielt [11].

Die Problematik der Fügetechnik [12] und der Krafteinleitung in metallische Schäume [13] sowie die Bearbeitbarkeit mit spanabhebenden Verfahren [14] wurde in fertigungstechnologisch orientierten Untersuchungen zur Anwendung in komplexen Bauteilen behandelt.

Eine weitere Erhöhung der Porosität auf bis zu 95\% (und damit zugänglichen Leichtbauwerkstoffen auf Eisenbasis mit Dichten bis hinab zu $0,2 \mathrm{~g} / \mathrm{cm}^{3}$ ) gelingt mit metallischen Hohlkugelstrukturen, die nach einem erprobten Verfahren von Polystyrolkugeln abgeformt und gesintert werden können [15].

\section{Aufgabenstellung}

Die Herstellung von geschäumten Keramikformkörpern kann über die Zwischenstufe geschäumter PolyurethanSysteme (PUR) erfolgen, die keramischen Pulver als Sintermaterial enthalten. Dies beruht auf der Annahme, dass PUR-Systeme mit bis zu 600\% ihrer Masse mit anorganischen Stoffen beladen werden können und dabei schäumbar bleiben. Die Polyurethane bzw. deren Schäume weisen einerseits ein weit einstellbares Eigenschaftsspektrum auf, andererseits dienen sie lediglich der Strukturierung des Schaumkörpers und werden anschließend ausgebrannt - sie sind letztlich nicht mehr im Produkt enthalten. Der Einsatz von PUR-Systemen auf der Basis von Recyclingpolyolen ist besonders interessant, da relativ preiswerte Polyole genutzt werden können. Folglich bestand ein Aspekt der Aufgabe in der Entwicklung und Rezeptierung der erforderlichen Polyurethan-Systeme, in der die Polyol-Komponente (A-Komponente) des Polyurethansystems angepasst werden musste.

Die als Hochleistungsmaterial einzustufenden Schaumkeramiken können nach diesem Verfahren mittels direkter Schäumung hergestellt werden (Abb. 1b), wobei die als Zwischenstufe entstehenden organischanorganischen Schaumstoffe mittels spezieller Temperaturprogramme in Brennöfen ausgebrannt und zu keramischen Schaumstoffen gesintert werden. Als anorganische Füllstoffe kommen in erster Linie keramische Pulver in Frage, deren Sinterfähigkeit bei Temperaturen um $1100^{\circ} \mathrm{C}$ einsetzt indem an sie den Oberflächen der Pulverkörner verschmolzen werden.

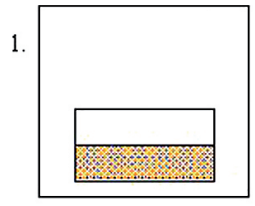

Schlicker

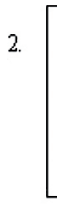

mit Schaumstoff (Schwamm) aufnehmen
Abb. 1a: Erzeugung fester Schaumstoffe durch Abformung einer Schaumstruktur

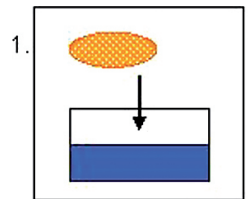

Keramikpulver

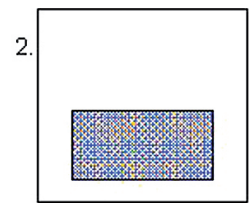

Schäumung

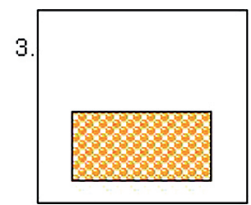

Ausbrennen in Polyol oder A-Komponente

Abb. 1b: Verfahren zur Erzeugung keramischer Schaumstoffe mittels direkter Schäumung

Offenzellige Schaumkeramiken geringer Wärmeleitfähigkeit eignen sich auch als Trägermaterial für Katalysatoren, wobei durch die Verwendung von aufgemahlenen harten Schlacken und Recyclatkeramik eine hohe Festigkeit in die Schaumkörper gebracht werden kann.

Die meisten der in Automobilen eingesetzten Katalysatoren besitzen keramische Wabenkörper als Trägerkörper für die eigentlich katalytisch aktiven Substanzen. Diese Trägerkörper werden zurückgewonnen, indem der Konverter (umgangssprachlich wird zumeist das gesamte Bauteil als Katalysator bezeichnet) mit einer hydraulischen Schere aufgeschnitten, und der Monolithbruch herausgenommen wird. Bei dem Monolith handelt es sich um hochwertige Keramik auf der Basis von Cordierit $\left(\mathrm{Mg}_{2} \mathrm{Al}_{4} \mathrm{Si}_{5} \mathrm{O}_{18}\right)$. Diese wurde bei der Herstellung der Katalysatoren mit einer Suspension (Slurry) aus Aluminiumoxid, seltenen Erden (in Oxidform), Übergangsmetallen und den katalytisch aktiven Elementen Platin, Palladium und Rhodium beschichtet. Diese Beschichtung macht ca. $10 \%$ der Gesamtmasse des beschichteten Trägers aus, die Edelmetalle alleine sind in Konzentrationen von ca. $2000 \mathrm{mg} / \mathrm{kg}$ (ppm) enthalten.

Zur Rückgewinnung der Edelmetalle wird dieser Keramikbruch an Scheideanstalten gegeben. Der gegenwärtige Stand der Technik ist, dass das edelmetallhaltige Material dort mit einem Sammlermetall $(\mathrm{Cu}, \mathrm{Fe})$ und einigen $\mathrm{Zu}$ schlagstoffen verschmolzen wird. Dabei entsteht eine Legierung des Sammlermetalls mit den Edelmetallen und eine keramische Schlacke, die gegenwärtig noch deponiert wird. Alternativverfahren gehen davon aus, die Edelmetalle mittels Säuren aus der Keramik herauszulösen (hydrometallurgische Verarbeitung). Mit der Umsetzung der zurückbleibenden gebrochenen Cordieritkeramik, die nach Aufbereitung wieder zu keramischen Material gebrannt werden können, setzen die Untersuchungen an.

\section{Ergebnisse}

Ausgehend von vielfältigen Erfahrungen in der Darstellung von metallischen Schäumen durch das Tränken eines Weichschaums mit einem metallpulverhaltigen Schlicker zur Erzeugung von Grünkörpern (siehe Abb. 1a) und anschließendem Verdampfen der polymeren Matrix wurde diese Methode auf keramische Pulver auf Basis von cordieritbasierten Katalysatorträgermaterialien übertragen. Wenngleich beide Verfahren (siehe Abb. 1) durch Brennen eines Grünkörpers zu Schaumkeramiken gelangen, so ist ersichtlich, dass die ggf. eigenschaftsbestimmende Morphologie der erhaltenen Produkte verschieden ist. 


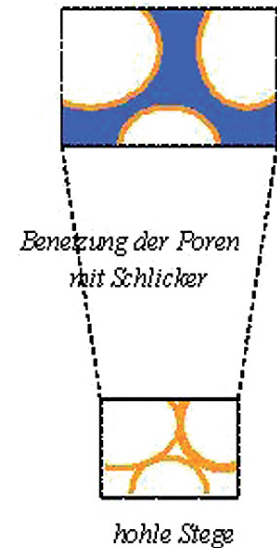

hohle Stege
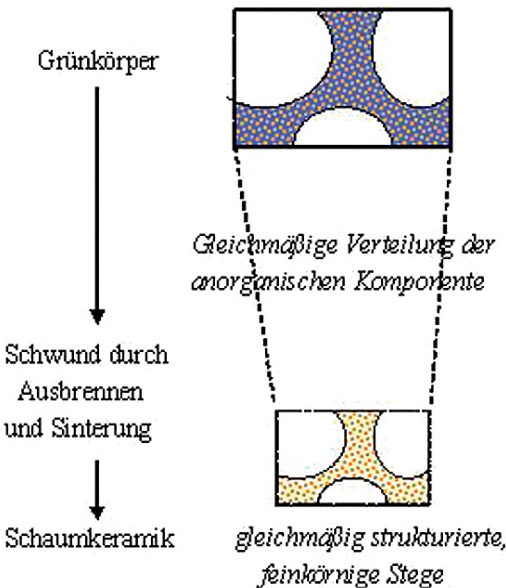

Abb. 2: Abhängigkeit der Mikrostruktur der Schaumkeramik vom Herstellungsprozess

Somit ist nachvollziehbar, dass über die Beherrschung der technologischen Schritte die Materialeigenschaften gezielt eingestellt werden können. Von geschlossenzelligen Schaumkeramiken ist bei gleicher Dichte eine höhere Festigkeit, aber verringerte Gasdurchlässigkeit gegenüber offenzelligen Schaumstoffen zu erwarten gewesen. Die Wärmeleitfähigkeit wird maßgeblich durch die Stegdicke und Körnigkeit bzw. Morphologie bestimmt, während für die Isolierwirkung, d. h. den mit der Gasdurchlässigkeit erfassten konvektiven Wärmetransport, auch die Zellstruktur (Vakuum oder luftgefüllt, Reflexion von Wärmestrahlung) maßgeblich ist.

Die folgende Tabelle enthält Rezepturbeispiele für PUR-Schaumstoffe, deren Beladung mit keramischen Pulvern erfolgreich durchgeführt wurde. Die auf diesem Wege hergestellten Hartschaumstoffe eignen sich als Matrix zur Einbettung keramischer Pulver. Ihre Zelligkeit kann zu Schaumkeramiken mit einer analogen Makrostruktur gebrannt werden.

\begin{tabular}{|l|l|l|l|l|}
\hline \multirow{2}{*}{} & $\begin{array}{l}\text { Substanz, } \\
\text { Mengenangaben in } \\
\text { Gramm }\end{array}$ & $\begin{array}{l}\text { PUR- } \\
\text { Recyclat }\end{array}$ & $\begin{array}{l}\text { PET- } \\
\text { Recyclat } \\
\text { P-185 }\end{array}$ & $\begin{array}{l}\text { PET- } \\
\text { Recyclat } \\
\text { LF027 }\end{array}$ \\
\hline $\begin{array}{l}\text { A- } \\
\text { Kompo- } \\
\text { nente }\end{array}$ & Polyol & 172,5 & 153,7 & 25 \\
\cline { 2 - 5 } & Dimethyletanolamin & 0,5 & 0,6 & \\
\cline { 2 - 5 } & $\begin{array}{l}\text { Dimethylcyclohexy- } \\
\text { lamin }\end{array}$ & & 0,3 & \\
\cline { 2 - 5 } & Polycat NP-40 & 0,3 & 0,2 & 0,1 \\
\cline { 2 - 5 } & Tegostab 8433 & & 0,05 \\
\cline { 2 - 5 } & Tegostab-B & 0,4 & 0,5 & 1 \\
\cline { 2 - 5 } & Wasser & 4,5 & 4,5 & \\
\hline \multirow{2}{*}{$\begin{array}{l}\text { K- } \\
\text { nompo- }\end{array}$} & $\begin{array}{l}\text { Lupranat M 20 A } \\
\text { NCO-Gehalt 31,8 \% }\end{array}$ & 202,5 & 200,0 & 30 \\
\hline & Rührzeit [s] & 10 & 10 & \\
\hline & Startzeit [s] & 16 & 18 & \\
\hline & Steigzeit [s] & 68 & 72 & \\
\hline & Abbindezeit [s] & 90 & 94 & \\
\hline
\end{tabular}

Neben der zu beachtenden Stöchiometrie der Hauptbestandteile stehen mit dem Katalysatorsystem, dem Treibmittel und dem Schaumstabilisator drei variable Parameter zur Steuerung der PUR-Schaumbildung zur Verfügung.
Die Auslegung der Brennprogramme basiert auf den thermischen Analysen (DSC) der mit Feststoffen beladenen PUR-Schaumstoffe. Hierdurch lassen sich die Temperaturbereiche, in denen die Umsetzungen erfolgen recht genau bestimmen und entsprechende Zeitintervalle für den Brennprozess definieren.

Mit dem Brennprogramm wird zuerst die Ausgasung der organischen Matrix gesteuert. Hierbei wird mit moderaten Aufheizgeschwindigkeiten einer Zerstörung der Schaumstruktur durch entweichende Gase begegnet. Mit den zwischenzeitlichen Haltephasen (ohne Temperaturanstieg) wird in den mittels der thermischen Analysen als Phasen besonders starken Stoffumsatzes ermittelten Temperaturbereichen eine schonende Umsetzung gewährleistet.

Für größere Formkörper sind gegebenenfalls längere Brenndauern bzw. langsamere Aufheizgeschwindigkeiten erforderlich. Eine ausreichende Brenndauer bei Sintertemperatur und eine kontrollierte Abkühlung tragen zur Vermeidung innerer Spannungen bei.

Die Schmelztemperatur der Schlacken aus dem pyrometallurgischen Refining-Prozess von KFZ-Abgaskatalysatoren wurde iterativ zu ca. $1150^{\circ} \mathrm{C}$ ermittelt, d. h. sie ist deutlich gegenüber der Schmelztemperatur von Cordierit $\left(1475^{\circ} \mathrm{C}\right)$ verringert und liegt noch beträchtlich unter dem angewandten Temperaturbereich (1250 - $1400^{\circ}$ C) für die Sinterung des Cordierit.

Die optische Charakterisierung erfolgte anhand von lichtmikroskopischen und elektronenmikroskopischen Aufnahmen. Die lichtmikroskopischen Aufnahmen dienten vor allem der Charakterisierung der Zelldichte, einem für eine Nutzung als Katalysatorträger maßgeblichen technischen Parameter. Mit den elektronenmikroskopischen Aufnahmen gelingt es, die Porosität im Festkörper selber zu erfassen.

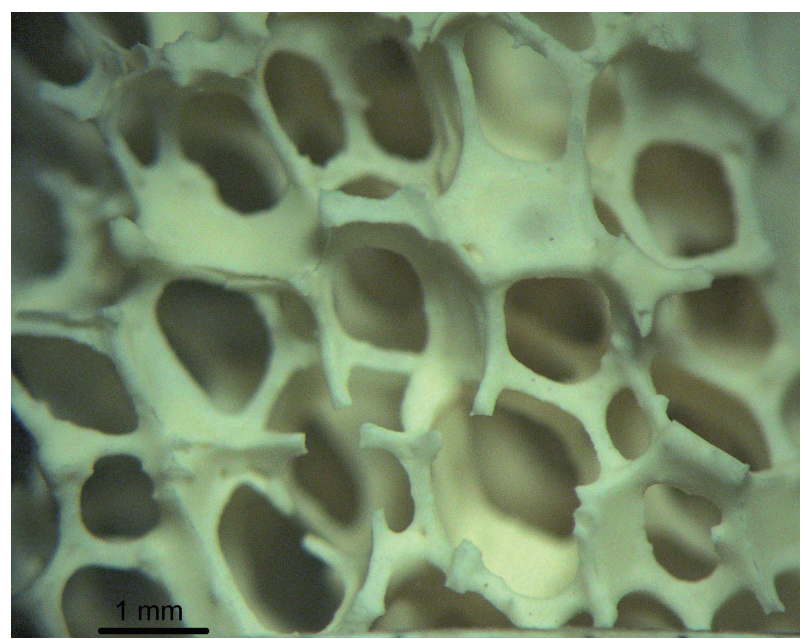

Abb. 3: Lichtmikroskopische Aufnahme einer Schaumkeramik, deutlich sichtbar sind die holen Stege, die durch das Abdampfen der PUR-Matrix entstanden sind. 


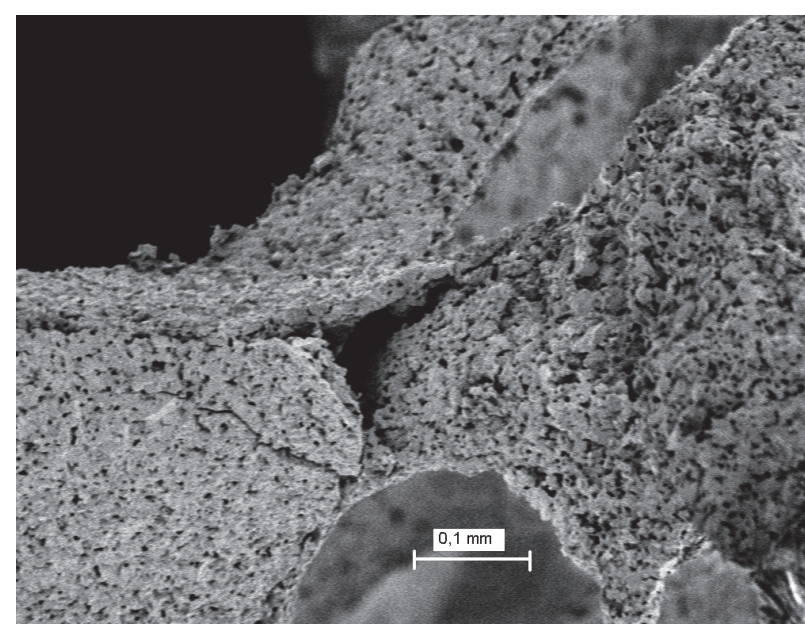

Abb. 4: Elektronenmikroskopische Aufnahme einer Schaumkeramik an einer Bruchfläche, die Körnung und Porosität sind gut erkennbar.

Es wurden die Druckverluste von Schaumkeramiken unterschiedlicher Porosität und Materialstärke in Abhängigkeit von der Durchströmungsmenge ermittelt. (Abb. 5) Als Vergleichsgröße wurde ein typischer Cordieritkeramik- Katalysatorträger mit Wabenstruktur und einer Zellenzahl von 400 cpsi (cells per square inch) gemessen.

Folgende Druckverluste wurden für die hergestellten Keramikschäume beobachtet:

10 ppi-Schaum ca. $0,025 \mathrm{mbar} / \mathrm{cm}$

20 ppi-Schaum ca. $0,05 \mathrm{mbar} / \mathrm{cm}$

30 ppi-Schaum ca. $0,1 \mathrm{mbar} / \mathrm{cm}$

Im Vergleich dazu liegt der Druckverlust für die Keramikwabenstruktur (400 cpsi) bei ca. 0,09 mbar/cm .

Die Werte zeigen, dass bis zu einer Porosität von 30 ppi das Keramikschaummaterial für den Einsatz als Katalysatorträger wegen seiner guten Durchströmbarkeit geeignet ist.

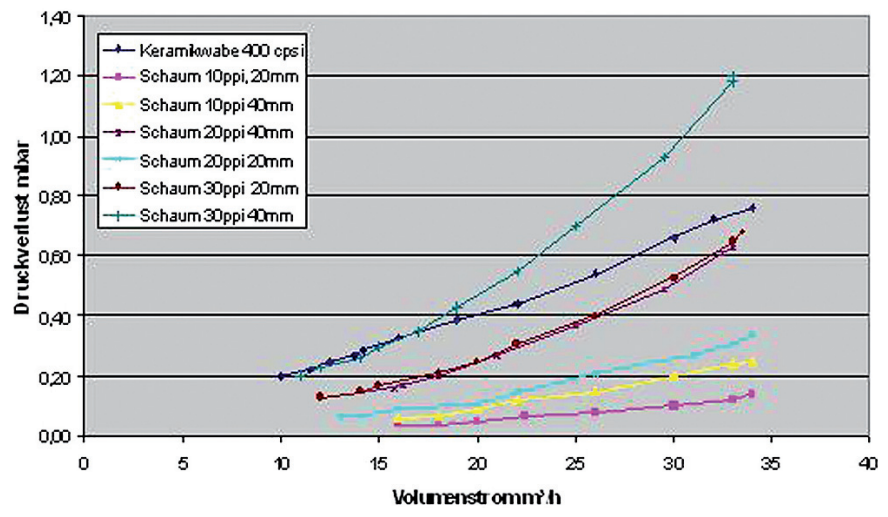

Abb. 5: Druckverlust-Vergleich Keramikwabe mit Schaumstruktur

Die Strömung durch eine Schaumstruktur führt aufgrund ihrer Geometrie zu einem wesentlich intensiveren Kontakt der Gasmoleküle mit der Oberfläche als bei der Durchströmung eines Kanals, wie er bei der Wabenstruktur vorliegt, d. h. die katalytische Aktivität wird durch den häufigeren Kontakt der Gasmoleküle mit dem Katalysatormaterial wesentlich erhöht.

\section{Danksagung}

Der Dank gilt dem Bundesministerium für Forschung und Technologie für die Unterstützung der Untersuchungen unter dem Förderkennzeichen 03 I 0209 B im Rahmen von Innoregio FIRM (01.10.04 - 30.06.06) und dem Kooperationspartner ReMetall Drochow GmbH für die anwendungsorientierten Beiträge im Verbundvorhaben.

Im Rahmen des Wettbewerbs »Effiziente Kooperationen zwischen Hochschulen und Unternehmen/Verwaltungen « aus Anlass der Veranstaltungsreihe der TFH Wildau »15 Jahre zuverlässiger und erfolgreicher Partner der Wirtschaft und Verwaltung « erreichte das Projekt »Entwicklung von geschäumten Keramikformkörpern unter Verwendung von Recyclingpolyolen«am 27. April 2007 den zweiten Platz.

\section{Referenzen}

[1] Scheffler, M./Colombo, P., Cellular Ceramics: Structure, Manufacturing, Properties and Applications, WileyVCH, Weinheim 2005

[2] Gibson, L. J./Ashby, M. F., Cellular Solids: Structure and Properties, Cambridge University Press 1997

[3] Svinka, V./Mörtel, H./Krebs, S. , DE 10134524

[4] Hing, K./Bonfield, W., EP 1117626

[5] Händle, F., DE 19613783

[6] Adler, J./Teichgräber, M./Standke, G./Jaunich, H./Stöver, H./Stötzel, R., EP 0907621

[7] Behrendt, G. et al., DE 19963554

[8] Fraunhofer IKTS Dresden; Adler, J. /Teichgräber, M./ Standke, G., DE 19621638

[9] Zeschky, J. et al., Zeitschrift für Metallkunde, 93 [8], 812 (2002)

[10] Degischer, H. P. in: Metallische Verbundwerkstoffe, K.U. Kainer (Hrsg.), DGM Informationsgesellschaft, Oberursel 1994

[11] Zeschky, J./Neubauer, J./Lo, J./Scheffler, M./Kummer, B./Greil, P., in: H.P. Degischer (Hrsg.): Verbundwerkstoffe, Wiley-VCH 2003

[12] Bach, F. W./Balzer, H./Kruzhanov, V./Zeitz, V., ZMW 2001

[13] Banhart, J./Baumeister, J./Weber, M., Sonderausgabe von ATZ und MTZ, Werkstoffe im Automobilbau $97 / 98$

[14] Denkena, B./Friemuth, T./Boehnke, D., Proceedings of Materials Week 2002, 436

[15] Stephani, G., Superleichte metallische Hohlkugelstrukturen in: Tagungsband WING-Konferenz 2003, 172 ff. PTJ 2003 


\section{Literatur}

Adler, J./Teichgräber, M./Standke, G./Jaunich, H./Stöver, H./ Stötzel, R., EP 0907621

Adler, J./Teichgräber, M./Standke, G., DE 19621638

Bach, F. W./Balzer, H./Kruzhanov, V./Zeitz, V., ZMW 2001

Banhart, J./Baumeister, J./Weber, M., Sonderausgabe von ATZ und MTZ, Werkstoffe im Automobilbau 97/98

Behrendt, G. et al., DE 19963554

Degischer, H. P., in: K. U. Kainer (Hrsg.), Metallische Verbundwerkstoffe, DGM Informationsgesellschaft, Oberursel 1994

Denkena, B./Friemuth, T./Boehnke, D., Proceedings of Materials Week 2002

Gibson, L. J./Ashby, M. F., Cellular Solids: Structure and Properties, Cambridge University Press 1997

Händle, F., DE 19613783

Hing, K./Bonfield, W., EP 1117626

Scheffler, M./Colombo, P., Cellular Ceramics: Structure, Manufacturing, Properties and Applications; WileyVCH, Weinheim 2005

Stephani, G., Superleichte metallische Hohlkugelstrukturen in: Tagungsband WING-Konferenz 2003, PTJ 2003

Svinka, V./Mörtel, H./Krebs, S., DE 10134524

Zeschky, J. et al. in: Zeitschrift für Metallkunde, 93 [8], 812 2002

Zeschky, J./Neubauer, J./Lo, J./Scheffler, M./Kummer, B./ Greil, P. in: H. P. Degischer (Hrsg.), Verbundwerkstoffe, Wiley-VCH 2003

\section{Autoren}

\section{Dr. rer. nat. Michael Herzog}

Technische Fachhochschule Wildau

FB Ingenieurwesen/Wirtschaftsingenieurwesen

Tel. +49 3375 508-332

michael.herzog@tfh-wildau.de

\section{Dr. rer. nat. Rainer Wegner}

ReMetall Drochow GmbH

Hauptstr. 2a

01994 Drochow

Tel. +4935754 749-27

rwegner@ecom-parts.com 